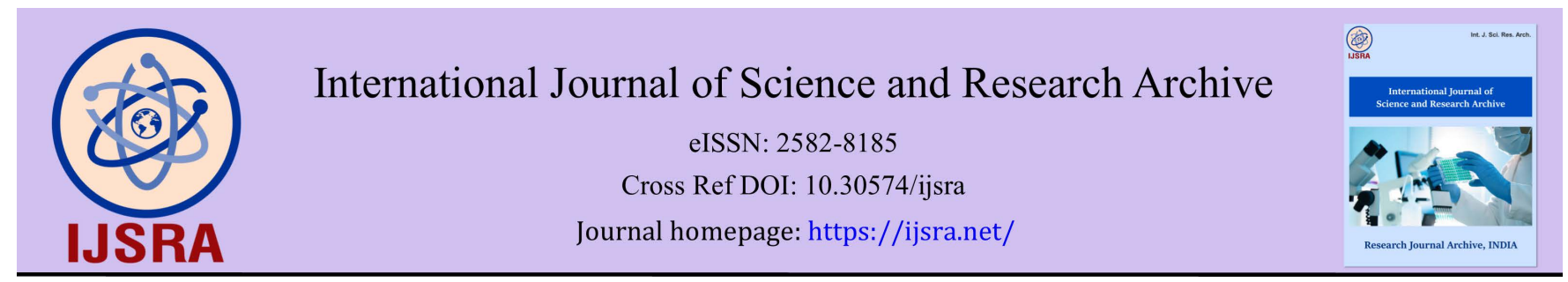

(REVIEW ARTICLE)

\title{
Interface between orthodox and traditional medicine and alternative paradigm for integrating orthodox and traditional health care in Nigeria
}

\author{
Amole 0.0. * \\ Lagos State University, College of Medicine, Department of Pharmacology, Therapeutics and Toxicology, Ikeja, Lagos. \\ Nigeria.
}

International Journal of Science and Research Archive, 2021, 02(01), 079-084

Publication history: Received on 10 January 2021; revised on 11 February 2021; accepted on 13 February 2021

Article DOI: https://doi.org/10.30574/ijsra.2021.2.1.0022

\begin{abstract}
Traditional medicine refers to the knowledge, skills and practices based on the theories, beliefs and experiences indigenous to different cultures, used in the maintenance of health and in the prevention, diagnosis, improvement of physical and mental illness. Herbal treatments are the most form of traditional medicine and $70-80 \%$ of the African region has used a form as primary health care. There is little doubt that the use of herbal medicines is growing and one of the most problems found in medicinal plants is the absence of clinical, toxicological and pharmacological studies. Many African countries depend on herbal medicines because of their efficacy, affordability and availability. The future of African traditional medicine is bright if view in the context of service provision, increase of health care coverage, economic potential and poverty reduction. Formal recognition and integration of traditional medicine into conventional medicine would be of good advantage.
\end{abstract}

Keywords: Traditional; orthodox; medicine; health care; integration.

\section{Introduction}

Traditional medicine is a rather vague term loosely used to distinguish ancient and culture-bound health care practices which existed before the application of science to health matters in official modern scientific medicine or allopathy. Some frequently used synonyms are indigenous, unorthodox, alternative, folk, ethno, fringe and unofficial medicine and healing. The term is unsatisfactory because it implies broadly that there is some body of principle, knowledge and skills common to all the varieties of traditional medicine, and because it does not distinguish between all-embracing and complex systems health care such as Ayurveda on the one hand and simple home remedies on the other [1].

Traditional medicine has been practiced to some degree in all cultures and other terms based on culture include African, Asian or Chinese medicine. Traditional practitioners define life as "the union of body, senses, mind and souls" and describe positive health as "the blending of physical, mental, social, moral and spiritual welfare". The moral and spiritual aspects of life are here stressed, thereby giving greater dimensions to the system of health care by which man maintains health [1].

How countries can make health and medical care available to all their citizens and communities has been one of the major preoccupations of politicians, administrators, the community and healthy workers. Since WHO's creation in 1948, debates in the World Health Assembly in the Regional Committees and in technical meetings bear witness to this concern and reflect the ambitions, the frustrations and the controversial nature of some problems to which such concern gives rise. A new sense of urgency has characterized recent discussions, which have culminated in the global resolve to accomplish a total health and medical care coverage for all people [1].

\footnotetext{
* Corresponding author: Amole, 0.0. Telephone: +2348033327658; Email: femiamole@yahoo.co.uk

Lagos State University, College of Medicine, Department of Pharmacology, Therapeutics and Toxicology, Ikeja, Lagos. Nigeria.

Copyright (C) 2021 Author(s) retain the copyright of this article. This article is published under the terms of the Creative Commons Attribution Liscense 4.0.
} 
Until the beginning of the $19^{\text {th }}$ century all medical practice was what we now call traditional. It was then that the great philosophical upheaval of the renaissance began to introduce Cartesian scientific materialism into all human activities and notably into the theory and practice of health care. The new way of looking at things subjected all assumptions to experiment and statistical validation and foresaw the future in terms of research and organization of necessity introduced doubt where previously there had been belief; it emphasized intellect and logic and belittled emotion and intuition. Its method was to break up complex phenomena into their component parts and deal with each one in isolation [1].

In diagnosis, this approach resulted in a search for a single cause, in pharmacology the search was for an active principle that could be isolated, and in the doctor-patient relationship the search for an efficient treatment of the physical cause of symptoms tended to exclude any serious interest in the complexity of the life situation in which the patient was immersed [2]. The application of scientific method to medicine and public health brought dramatic improvements in all those conditions in which material factors such as infection, poisoning, injury, nutrition or personal and environmental hygiene play a major part in the etiology. In degenerative conditions, however, the results have been less striking, and in conditions where behavioural, emotional or spiritual factors play a major role it would be difficult to argue that the scientific method has produced noticeable improvement; some would contend that deterioration is evident. Since psychosomatic disturbance is today one of the commonest of human afflictions, the philosophy and functioning of modern health and medical services is being questioned in many quarters. Add to this is the fact that only in richest countries has the modern allopathic health service been able to achieve a significant coverage of the population-at what is becoming an intolerable cost - and the search for alternatives seems highly topical [3].

\section{The role of traditional medicine in primary health care}

Primary health care is essential health care based on practical, scientifically sound, socially acceptable methods and technology. The principles that are necessary for its effective deployment require a multisectoral approach as determined by various activities and facilities. It is the main vehicle through which an acceptable level of health and near total coverage can be achieved in the foreseeable future. Primary health care is concerned with the main health problems in the community and the services reflect the political and socioeconomic patterns in the country. In order to make such care readily accessible and acceptable in the community, maximum self-reliance and community participation for health development are essential. Such involvement enables communities to deal with their health problems in the most suitable ways and community leaders are in a better position to make rational decisions concerning primary health care and to ensure appropriate support for health and allied projects. Primary health care workers should whenever applicable, include the traditional practitioners-healers, birth attendants etc. [4].

The developed countries are in a more favourable position, as on the whole their facilities and health manpower are both reasonably adequate. What is now required in some developed countries is a redistribution of functions and responsibilities, and more efficient restructuring to reduce costs and at the same time increase efficiency and productivity. In many countries, the soaring costs of health services have become extremely burdensome to the community. The developing countries like Nigeria, however, pose a picture of want and deprivation with inadequate resources, a dearth of manpower, and no definite hope of amelioration in the for seeable future except through the adoption of unorthodox measures such as the exploitation of useful traditional health practices. This includes a wider use of locally produced herbal medicines and the incorporation of traditional practitioners into the health team [5].

The Traditional Medicine Programme, which was initiated some years ago, has focused on methods of evaluating medicinal plants and the training of traditional practitioners in the various aspects of primary health care. Perhaps the most important function of government in this connection is to ensure that traditional medicine is neither blindly endorsed nor rejected but is examined carefully with an open mind [6]. The use of medicinal plants in therapy is one of the most important components of traditional medicine and yet this use has remained virtually unexplored in scientific terms [7]. Many valuable drugs have been derived from plants, and information that a plant is utilized in traditional medicine still provides an indication that it is worth scientific study. Medicinal plants are commonly available in abundance, they can provide safe, stable, standardized, and effective galenical products for use in primary health care, and they can lead to the discovery of new biologically active plant-derived principles that may have a therapeutic potential encouragement and support given now to local studies on traditional medicinal plants, could well enable expenditure on imported drugs to be reduced, and thus promote economic self-reliance [7]. In addition, one of the chief contributions that traditional medicine has made and continues to make to health is the discovery of plants of medicinal value. "Save Plants that Save Lives" is a call to safeguard this heritage, and regulations should therefore cover conservation measures. The general feeling is that the future of traditional medicine is bright because they are widely used and respected, especially by the rural population that constitute the majority. Costs are also considered to be low. It is also obvious that specific legislation is needed in the country to recognize and legitimize traditional practitioners. 
The traditional practitioners should constitute themselves into an association through which they could interface with the formal system whether they are part of it or not [8]. An association of this nature could be a regulatory body in relation to ethical and professional matters. Without this formal structure, the chaos that exists now is likely to continue. Furthermore, it is imperative that steps be initiated to record and inventory all herbal remedies that are used in the country before the total disappearance of indigenous plant populations that often hold the key to finding new drugs that may benefit the global community. The establishment of their safety, based on published data and/ or preclinical scientific studies, should precede the use of manufactured medicinal plants for both self-medication and in national health services. When quality control has been assured, studies for efficacy could then be initiated [8].

There is widespread disenchantment with health care in the country, health resources tend to be concentrated in urban areas, which accommodate only about $20 \%$ of the total population - These facilities are so expensive that only the elite and opulent citizens in the city can afford to avail themselves of the specialist services utilizing such technology. In the final analysis, only a very small proportion of the total populations have ready access to such facilities, which absorb a major part of the total health budget. Meanwhile, orthodox and conventional health care services remain culturally unacceptable in some parts of the country and economically unobtainable.

The national average physician to population ratio was $1: 10,000$, over $80 \%$ of the nearly governmental salaried physicians work in the towns and cities, which accommodate $14 \%$ of the total population. The physician to population ratio for the rural areas is therefore in the region of 1:100,000. It is therefore not surprising that the rural-based indigenous healers should assume so much responsibility for the community's primary health care. In Nigeria, primary health care devolves on the healer, herbalist, traditional midwife and other traditional practitioners. These are the health workers who offer services to the disadvantaged groups that total $80 \%$ of the world's population and have no easy access to any permanent form of health care. Traditional medicine therefore has a major role in primary health care in terms of numbers of people served by that health care system throughout the world and in spite of any defects. The traditional practitioners are the true community health workers in their society. They invariably have the confidence of the people, and whatever their level of skills, it is essential that they should understand the real health needs of their community. There are, however, certain major constraints regarding the use of traditional medicine in primary health care, these are not insurmountable and, with goodwill on all sides, they can be overcome. The incorporation of traditional health systems into the official health system requires a major policy decision at the very high governmental policy.

\section{Drug development stages for traditional medicine}

Discovery: The discovery of a traditional medicine product is usually the combined effort of a botanist, a chemist and a pharmacologist. The defining of all the characteristics of a traditional product begins with the plant and usually ends with the identification of some active compounds or markers to be able to chemically characterize the traditional medicine product.

Basic Scientific Characterization. This becomes a very difficult problem when traditional medicines are being developed. The active principles may become the major target for identification by both chemical and biological methods. The primary question that must be asked is, to what level will the scientific characterization be carried? If the activity is found to be associated with a particular chemical, then should that agent be developed as purified drug? Since the basis for traditional medicine is the simple use of plants or plant products, the track of development may take two paths. In the first, the characterization is only developed for quality control and to insure the activity of the traditional preparation. A second path is where the active ingredients is developed as a new medicine in modern terms, (i.e., a pure compound formulated into a modern preparation such as a tablet). The second path would take the activity out of the traditional medicine arena and would then subject it to drug development regulations and laws. The lack of laws regarding traditions medicine must be addressed if traditional medicines are to be developed and incorporated into a health care system.

The basic scientific characterizations may have the following components:

Chemical: Identify parent compound, develop analogs, pilot batch, scale up and quality assurance.

Biological: Model In vitro, in vivo, organ response, pharmacokinetics.

Safety evaluation (Animal Studies). Traditional medicines are assumed to be safe. However, there is no large information base on the toxicology of these preparations. Used under normal conditions, there may be no immediate identifiable toxic events. However, it is difficult, to identify toxic events if the events are not associated with the period 
of time the traditional product is being administered. Some examples are carcinogencity and organ damage. The cancer or organ damage may be subclinical or progressive in development, such as in kidney diseases and therefore only manifest long after the traditional medicine was utilized. It will be critical to perform scientifically valid toxicology testing on traditionally medicine products to identify, delayed toxic events. The traditional medicine products being developed will be identified as pharmacologically active preparations. There is always a potential for excessive dosages or hypersentivity to a drug. Understanding the toxicology of traditional products would greatly assist in identifying individuals utilizing the preparation in excessive quantities and also help the clinician faced with accidental ingestion by defailing the symptoms of intoxication. There is a great need for toxicology testing prior to traditional medicine products being incorporated into and accepted as a part of an established health care program, which would support their development and distribution to large number of people. Some tests that should be performed are: acute toxicity, subchromic toxicity, chronic toxicity including carcinogenicity, reproductive effects including fertility, peri-and post natal effects and teratology, mutagenicity and carcinogenicity. It is recognized that the cost of toxicity testing is great and one of the primary purposes of a traditional medicine is the low cost of development. The burden of paying for the toxicology testing is a difficult problem that the government must deal with when considering its national policy dealing with traditional medicine development. Since the traditional medicines are not subject in most cases to patent protection, it would be difficult to convince commercial firms to bear the cost of the toxicology testing.

Formulation development. There are many questions in this area that relate to traditional medicines. The formulation may be as simple as determining the appropriate amount of material to be placed in a tea bag or whether to incorporate more than one part of the medicinal plant or a mixture of herbs. In many cases, some of the components of the traditional medicine may be present for their aroma and without pharmacological action. The development of a traditional medicine may cause the product to be used a different form. This then raises the questions of accept ability to the general population, which may prefer the traditional method of preparation. The aroma of a decoction may be an important part of the confidence the patient has in the activity of a medicine when compared to taking a compressed tablet that cooks and acts like a soft stove. Such questions must be addressed during formulation.

Human Pharmacology. There are a large number of traditional medicines being utilized. It would be essential to determine which of the traditional medicines would be the most effective with the least liability for side effects or toxicity. This would require carefully controlled clinical trials of the formulated products to evaluate potency, efficacy and safety. The clinical trials should be performed with careful design and observations in a similar manner to those carried out for modern drug testing. The proper design and performance of a clinical trial would be critical to gain the support of all the health care practitioners including modern medicine The design of a clinical trial for a traditional medicine must be tailored to the preparation. Some problems that must be addressed would be how to mimic aromas of the traditional medicine in order to have the clinical trial blind to the participant, or how to disguise the leaf/preparation in order for the participant to not know when the actual preparation was being administered. The development of clinical trials for traditional products would be a difficult but necessary task.

Regulatory process. There is a great need to identify a path to the registration and national acceptance of a traditional medicine preparation. Currently, there are little or no such paths. It is imperative that governments institute a process for the incorporation of traditional medicines into a national health care system. Without such legislation, there can be little control over which medicines are to be supported and the maintenance of good quality control over the products. The primary purpose would be to insure that only the active and safe preparations are being used and the products people have access to are proper in dosage and activity.

Marketing. The marketing of a traditional medicine will be important. The identification of a particular traditional medicine product that may be much more active than other traditional medicine that may be much more active than other traditional medicines or may be a good inexpensive substitute for a modern medicine would result in better and less expensive health care. However, the information must be disseminated to both the traditional practitioners and the general population. However, the dissemination of information to large numbers of people in primarily rural areas and in the poorest villages would be difficult - these would be special problems that must be addressed, since traditional medicines would not be protected by patents, the cost would probably be borne by the government.

Manufacture and supply. The ready availability of an identified safe and effective traditional medicine must be established. As in the marketing, there are costs involved in the development of manufacturing sites and establishment of supply mechanisms. Without patent protection it may be difficult to obtain private funding.

Post registration phase. The continued evaluation of a product should always be developed to insure safety and efficacy. Adverse side effects, drug interactions with other traditional medicines or modern medicines, and over use or about potential may be identified. 
Expanded markets. The development of a traditional medicine may lead to a product that would be exported to other countries.

\section{Supporting services}

When special care or advice on more difficult problems is required, the primary health care worker should have access to more highly trained health personnel with adequate facilities. The types of such personnel will vary according to resources, but they should have full appreciation of the various situations encountered at the primary health care level in order to be able effectively to handle problems referred to them. Their social and educational responsibilities in health education should be emphasized and their main educational function should be to teach the people how to look after themselves. Special attention should be paid to the mothers - "Educate a mother and you educate a family". Health workers could first learn what mothers believe and feel and then, mainly through practical demonstrations, help them to understand how they could improve their own and their family's health. Some serious thought will have to be given to no referral arrangements since without such back-up facilities the community would gradually lose confidence in their primary health care systems. Referrals should be carefully explained to both the patient and relatives because in several traditional societies, referrals to hospital is taken as an indication that the condition is hopeless and the patient is being sent to hospital to die. Patients do not care to be treated far away from their homes and whenever possible, health interventions should take place at community level. A clear explanation of those interventions and the technologies employed should always be given in simple language and preferably in the vernacular. Newspapers, films, radio and television could be employed for mobilizing community interest and support for the development of the primary health care programme. In communities where health committees are established for the implementation of health programmes it should ensure that not only government officers and educated persons serve on these committees but that community leaders, literate and illiterate, and senior traditional practitioners and midwives are also included as active participants. Their opinion should be sought on the initial planning, health priorities and overall implementation of the programme. It is mainly through such collaborative efforts involving some devolution of responsibilities coupled with delegation of authority that enthusiasm for community participation can be assured. Traditional practitioners can create a lot of difficulties at primary health care level when they feel, rightly or wrongly, that the administration is discriminating against them. "If you cannot beat them, join them".

\section{Conclusion}

The high cost of drugs and the inability of many individuals to purchase such drugs have prompted such individuals to look for local products in the form of medicinal plants and herbal medicines that have proved to be effective, safe, inexpensive and culturally acceptable. There are many records of traditional therapies employing herbal medicines that are said to be very effective against common ailments and usually without any side effects. A preliminary task would be to identify the most commonly used formulations for the treatment of common ailments in various localities. In certain Asian and Latin American countries, the cultivation of commonly used medicinal plants is undertaken in home and community gardens to ensure adequate and continuous supplies. This practice reduce the risk of extraction of endangered plant species. The cultivation of medicinal plants and herbs can also be conveniently linked with the production of vegetables and fruit with high nutritive value that should be of particular benefit to mothers and children. In the selection of drugs and herbal medicines for treatment, the health worker is expected to give preference to locally produce and equally effective preparations. Primary health care workers should have basic knowledge concerning medicinal plants, their cultivation, identification, collection and preparation for therapeutic applications within the community in which they work. The use of medicinal plants and traditional medicine could make people become more self-reliant. The year 1985 was a turning point in Finland, when a group of bonesetters and contemporary medical doctors of the same area began co-operating in their practices. This took place in Kaustinan, a locale of 4,000 inhabitants in the midwestern part of the country. The name of the town is renowned for another reason: Kaustinen Folk Music Festival. Subsequently, a Folk Medicine Centre was established in Kaustinen. Since folk medicine is a part of living ethnic tradition and the best aspects of it have always been appreciated in Finland, the activities of the Centre are financed to a large extent by the government. The main activities in the Folk Medicine Centre include the promotion of traditional Finnish folk medicine, scientific research and dissemination of information on folk medicine and research. In summary, one can say that the work here comprises health care, research and public relations activities. The proper development of a traditional medicine to be incorporated into a national health care system may be as costly as any modern drug. However, the potential benefits may also be great. It would insure the utilization of only the most active and safe traditional medicines. Also, the identification of a highly accessible, inexpensive substitute for a modern drug would provide greater accessibility to health care for economically disadvantaged individuals and reduce the tremendous outflow of hard currency. 


\section{Compliance with ethical standards}

\section{Acknowledgments}

The author acknowledges with gratitude, the World Health Organisation (WHO).

Disclosure of conflict of interest

No conflict of interest.

\section{References}

[1] World Health Organisation (WHO). Tradition medicine and health care coverage. 2nd Edition, Macmillan, England 1983. Pg $9-11$.

[2] Djuv A, Nilsen OG, Steinsbekk A. The co-use of conventional drugs and herbs among patients in Norwegian general practice: a cross-sectional study. BMC Complementary and Alternative Medicine. 2013; 13: 295 - 296.

[3] World Health Organisation (WHO). Tradition medicine fact sheet. No. 143; www.who.int/mediacentre/factsheets/fs134/en/accessed 15 November 2011.

[4] World Health Organisation (WHO). Alma.ata 1978. Primary health care. Alma-ata, USSR, 6 - 12 September 1978, Geneva, 1978 ("Health for All" series No. 1).

[5] Djukanovic V, mach EP, ed. Alternative approaches to meeting basic needs in developing countries: a joint UNICEF/WHO study. Geneva, World Health Organisation, 1975.

[6] Bannerman RH. Traditional medicine in modern health care services. Journal of the David Davies Memorial Institute of International Studies. 1980; 6(5): 731 - 747.

[7] Burkill HM. The useful plants of West Tropical Africa $2^{\text {nd }}$ Ed. Royal Botanic Gardens, Kew UK 2000; 5 - 686.

[8] National Health Planning Unit. An approach to planning the delivery of health care services. Accra, Ghana. Ministry of Health, 1979 (Manual No. 1). 